\title{
Lymphoma: turning biology into cures
}

\author{
Authors: Thomas Cummin ${ }^{A}$ and Peter Johnson ${ }^{B}$
}

Diffuse large B-cell lymphoma (DLBCL) is the commonest aggressive non-Hodgkin lymphoma with approximately 5,000 cases annually in the UK. The R-CHOP (rituximab, cyclophosphamide, doxorubicin, vincristine and prednisolone) regimen has become the international standard of care with cure rates of around $75 \%$ and despite extensive studies aimed at improving the outcomes, R-CHOP has not been superseded. Those patients that do not respond to R-CHOP have a poor outlook. DLBCL is a disease with marked molecular heterogeneity; advances in gene expression profiling and mutational analysis can be used to increase our understanding of the disease and identify new therapeutic targets. Precision medicine using new agents, including small molecule inhibitors, is now being investigated for DLBCL. Progress in this disease is likely to come by targeting heterogeneous subtypes through novel combinations. Where R-CHOP fails, we hope that these new approaches can succeed by providing personalised medicine using precision diagnostics to guide new treatment paradigms.

KEYWORDS: Cell of origin, diffuse large B-cell lymphoma, gene expression profiling, lymphoma, precision medicine

\section{Introduction}

Diffuse large B-cell lymphoma (DLBCL) is the commonest aggressive non-Hodgkin lymphoma, accounting for approximately 5,000 cases annually in the UK. There is a rising incidence, especially among those over the age of 65 years. For more than 25 years, the combination of cyclophosphamide, doxorubicin, vincristine and prednisolone (CHOP) chemotherapy was the standard treatment regimen, following which approximately half of patients experienced long-term remissions, equivalent to cure. In the early 2000s, the addition of the anti-CD20 monoclonal antibody rituximab improved response rates, event-free survival and overall survival for DLBCL patients; ${ }^{1}$ this R-CHOP regimen has since become the international standard of care with cure rates of around $75 \%$ in clinical trials. Despite extensive studies aimed at improving the outcomes, R-CHOP has not been superseded. Patients

Authors: ${ }^{\text {A }}$ clinical research fellow and specialist registrar in haematology, University of Southampton, Southampton, UK; B professor of medical oncology, University of Southampton, Southampton, UK alive without recurrence 24 months after diagnosis have an excellent prognosis, with similar life expectancy to age and sexmatched controls. ${ }^{2}$ However, up to one-third of patients have lymphoma that remains refractory or relapses, and these have a poor outlook. If they are fit enough, these patients are offered intensive salvage chemotherapy and autologous transplantation but this is only successful in about one fifth of cases.

More than half of patients diagnosed with DLBCL are over 60 years of age and while R-CHOP may be used in older patients, frailty and comorbidity limit the use of intensive chemotherapy in this cohort, resulting in suboptimal therapy and overall poorer outcomes. There is a substantial unmet medical need for new approaches with greater efficacy and less toxicity for those patients poorly served by R-CHOP. New techniques to characterise different subtypes of DLBCL, which may be amenable to different forms of treatment, and to identify new therapeutic targets represent an opportunity to improve clinical outcomes. Contemporary studies are using prospective molecular profiling and targeted new therapeutics to advance lymphoma treatment.

\section{Cell of origin}

DLBCL has historically been classified according to standard morphology under light microscopy and immunostaining, which the nomenclature reflects. The current classification does not explain why some lymphomas are immunochemotherapysensitive while others fail to respond. Molecular phenotyping using gene expression profiles and patterns of somatic mutation offers the possibility of addressing this deficit and thereby improving treatment. Investigators have discovered substantial molecular heterogeneity within the disease through these techniques. Gene expression profiling (GEP) is a technique using hybridisation of cDNA to arrays of short polynucleotides, representing the potentially expressed genes, to measure the relative expression of genes within a cell at the level of mRNA. GEP can include the whole transcriptome and measure thousands of genes, or can be used in more restricted microarrays to target the most informative transcripts. GEP has been shown to reflect a range of cellular functions in DLBCL and has identified at least three distinct subtypes. These are based on the point in B-cell ontogeny at which the transformation to malignancy has apparently occurred, the so-called 'cell of origin' (COO). The activated B-cell (ABC) subtype is associated with patterns of gene expression involved in B-cell receptor (BCR) signalling, specifically the NF- $\kappa \mathrm{B}$ pathway, while the germinal centre B-cell (GCB) type tends 
to express genes involved in the germinal centre reaction. A third type, primary mediastinal B-cell lymphoma (PMBL), has a different pattern of expression, which affects cytokine signalling pathways. The genes expressed in PMBL are strikingly similar to Hodgkin lymphoma, suggesting a possible COO from a subpopulation of thymic B-cells. ${ }^{3}$

Other less readily defined subtypes also exist, and a significant minority of DLBCL cannot be assigned to any of the three types described. Each subtype is also associated with preferential occurrence of mutations in the DNA, in genes such as $C D 79 B$ and MYD 88 in ABC and CREBBP and EZH2 in GCB types. Their molecular distinction is also reflected in some clinical heterogeneity. ABC-DLBCL is more likely to affect the central nervous system and is more common in older patients. Large retrospective studies suggest that the $\mathrm{ABC}$ type DLBCL has a worse prognosis with an overall survival of $40 \% .{ }^{4}$ However, this has not yet been proven in prospective studies, and other factors, eg chromosomal rearrangements or expression of genes such as $M y c$ and $B c l 2$, may be more influential. ${ }^{5}$ One retrospective subgroup analysis has suggested that GCB-DLBCL may show preferential responses to the R-DHAP (rituximab, dexamethasone, cytarabine and cisplatin) chemotherapy regimen. ${ }^{6}$

It has been suggested that $\mathrm{COO}$ might be used to select treatments targeted against specific aspects of lymphoma biology. This will require robust tests that can be readily applied in routine clinical practice. GEP to determine COO was first described over a decade ago, but the methods have taken time to optimise. ${ }^{7}$ The original arrays required fresh frozen biopsy material to obtain sufficient quantities of high-quality mRNA. Most DLBCL patients' diagnostic specimens are formalin-fixed biopsies and are often obtained by percutaneous needle core biopsy, which until recently has restricted the clinical utility of GEP. It is now possible to obtain reliable information from smaller samples by routine biopsy through advances in mRNA amplification and solid phase chemistry. GEP can now be performed on routine clinical samples more reliably.

GEP needs further refinement to determine the optimum technique and classifiers, and to demonstrate its value in the clinical environment on large cohorts of patients. The Affymetrix Lymphochip custom array was used by the Lymphoma Molecular Profiling Project, which selected 27 transcripts in an algorithm to determine COO. ${ }^{7}$ Other groups have developed their own algorithms to distinguish between subtypes, using different techniques to determine COO. There is, as yet, no universally accepted technique by which to subtype cases. Care et al's analysis has made some progress in identifying a common 20 -gene signature, ${ }^{8}$ which can be used to predict subtype accurately, even using different technologies for GEP. ${ }^{7}$ However, these techniques remain in the research domain and are not yet readily available in routine clinical practice. Immunohistochemistry (IHC), which is widely available, has been used as a surrogate to determine COO by a variety of algorithms in an effort to make this information accessible. The most widely adopted classifier (Hans) includes expression of CD10, Bcl-6 and Mum-1, which gives $80 \%$ concordance with GEP, while others have been suggested to improve accuracy. ${ }^{9}$ However, there is a significant problem of interoperator variability in results with IHC with poor levels of reproducibility, which makes GEP by some way the most accurate method in determining COO.

\section{Emergence of targeting}

The biology of DLBCL, based on understanding COO and genomic mutation profile, is just beginning to be used to select effective treatments. The NF- $\mathrm{BB}$ pathway can be targeted in $\mathrm{ABC}-\mathrm{DLBCL}$ by treating patients with selective agents. Bruton's tyrosine kinase lies upstream in the BCR signalling pathway to $\mathrm{NF}-\kappa \mathrm{B}$, and the inhibitor ibrutinib is the first drug that inhibits this kinase to receive a licence in B-cell malignancies. In a phase II trial of patients with relapsed/refractory lymphoma, 37\% with ABC-subtype showed objective responses to the drug compared with only $5 \%$ of GCB. ${ }^{10}$ Bortezomib is a proteasome inhibitor that may prevent degradation of I $\kappa-\mathrm{B}-$ an inhibitor of NF- $\kappa \mathrm{B}$ to which $\mathrm{ABC}$ cell lines show particular sensitivity. ${ }^{11} \mathrm{~A}$ small pilot study showed a higher response rate in $\mathrm{ABC}$ than $\mathrm{GCB}$ relapsedrefractory DLBCL. ${ }^{12}$ A large phase III trial involving bortezomib (REMoDL-B) with prospective molecular-profiling has completed recruitment of more than 1,100 patients and the results are awaited. ${ }^{13}$ There is evidence that adding lenalidomide - an immune-modulating drug that may downregulate NF- $\kappa \mathrm{B}-$ to $\mathrm{R}-\mathrm{CHOP}$ results in similar outcomes in lymphoma of $\mathrm{ABC}$ subtype as GCB. The investigators suggest this may be because it ameliorates the inferior outcomes normally seen in ABCDLBCL. ${ }^{14}$ As therapeutics act at different sites, molecularly-defined DLBCL subgroups may respond differently and studies will increasingly need to record this information and stratify according to the results.

The DLBCL genome has been widely studied to characterise the abnormalities that contribute to the disease process. Structural rearrangements due to breakpoints are sometimes present, most often including $\mathrm{Myc}, \mathrm{Bcl} 2$ and/or Bcl6 translocations detectable by cytogenetic analysis. There is extensive variation of mutations between cases, along with temporal and subclonal variation within cases. Individual cases may contain several mutations that can be acquired throughout the life cycle of the lymphoma. These include early mutations key to pathogenesis and later mutations that may drive clonal expansion or have no pathogenic effect. Driver mutations provide important targets for treatment, potentially changing the course of the disease.

\section{Recurrent mutations}

Within COO subtypes the frequencies of mutations vary, but each subtype shows characteristic mutation profiles. $\mathrm{ABC}$ clones tend to contain common mutations upstream of NF- $\kappa B$, such as $C D 79 A / B, C A R D 11$ or MYD88. While these are not exclusive to $A B C$, they are significantly more frequent in this subtype than others. ${ }^{15}$ Upstream mutations may provide drug resistance to proximally targeting therapeutics, such as ibrutinib or fostamatinib, and require an alternative approach (Fig 1). CARD11 mutations do appear to confer resistance to ibrutinib and sostaurin (a protein kinase $\mathrm{C}$ inhibitor) in the $\mathrm{ABC}$ subtype. ${ }^{10,15}$ However, there is evidence that concomitant MYD88 and CD79B mutations may be more responsive to ibrutinib. In vitro testing predicts that DLBCL with the CD79B mutation will respond favourably to sostaurin. ${ }^{15}$ The presence of BCR mutations and concomitant MYD88 mutation may predict response, but are not essential for responses to interruption of BCR signalling. ${ }^{10}$ In the ABC phenotype, specific mutations in TNFAIP3 and GNA13 appear to be associated with R-CHOP resistance and may be identified for an alternative approach in future. ${ }^{15}$ 


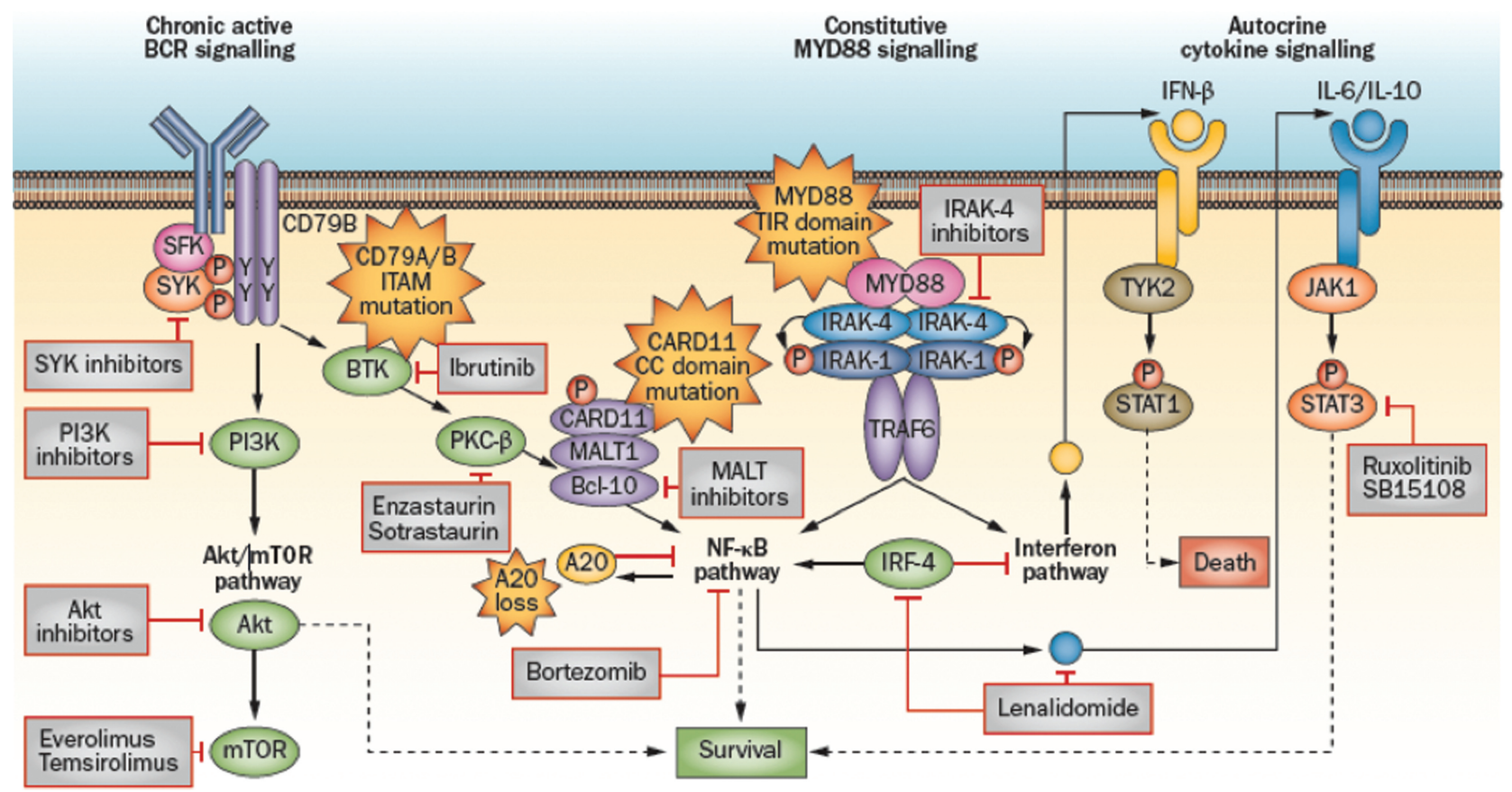

Fig 1. The key signalling pathways implicated in activated B-cell diffuse large B-cell lymphoma with targeted novel agents in clinical development. Reproduced with permission from Roschewski et al. ${ }^{4}$

The GCB-subtype appears less dependent on BCRsignalling, but molecular profiling provides alternative mechanisms that may be potential targets for therapy. The most frequent mutations include genes involved in epigenetic regulation and the cell cycle pathway. EZH2, CREBBP and EP300 are epigenetic regulators with mutational changes characteristic of GCB-subtype. EZH2, MLL2 and CREBBP have been found to be significantly more often mutated in GCB-DLBCL. ${ }^{15}$ A study of $42 \mathrm{ABC}$ and 83 GCB lymphomas found $22 \%$ of GCB had $E Z H 2$ point mutations; none were present in ABC cells. ${ }^{16}$ Like NF- $\kappa \mathrm{B}$ in ABC, EZH2 is thought to be pathogenetic in the GCB phenotype, silencing tumour-suppressor and anti-proliferative gene transcription. Regardless of gene mutations, GEP shows increased EZH2 expression in both GCB and ABC cell lines, where it is associated with aggressive disease. ${ }^{17} \mathrm{EZH} 2$ inhibitors have shown efficacy in pre-clinical studies by preventing proliferation of EZH2-mutant cell lines and suppressing growth of mutant $E Z H 2$ xenografts in mice. ${ }^{18}$ A separate study investigating cell growth in the presence of $E Z H 2$ inhibitors found that wild-type EZH2 cells and mutant cells of the GCB phenotype were both affected, but the mutants were more sensitive. Conversely, ABC-type cell growth was unaffected by $E Z H 2$ inhibitors. ${ }^{19}$ Novel EZH2 inhibitors are currently part of phase I and II clinical trials and have shown promising efficacy in patients with lymphoma resistant to multiple lines of therapy, mostly in the GCB-subtype. ${ }^{20}$

\section{'Double-hit' and 'double-expressers'}

It has been known for some time that rearrangement of the $M y c$ gene in DLBCL is associated with worse outcomes.
$M y c$ is a regulator oncogene that is rearranged in 5-15\% of DLBCL cases. ${ }^{21}$ Affected patients are more likely to experience treatment failure after R-CHOP treatment, and in this situation even myeloablative therapy is of marginal benefit. ${ }^{22} \mathrm{Co}$-existent rearrangements in $\mathrm{Bcl} 2$ or $\mathrm{Bcl} 6$ appear to compound the poor prognosis, with 'double-hit' cohorts demonstrating particularly aggressive disease. ${ }^{23} \mathrm{Bcl} 2$ has an anti-apoptotic role and confers increased survival to the malignant cells. Bcl6 is a transcription factor that suppresses genes involved in proliferation, survival and cell growth. $M y c / B c l 2$ double translocations are seen more often in the GCB phenotype. ${ }^{24}$ However, the partner gene involved in $M y c$ translocation may predict prognosis but only appears prognostic if high expression of $M y c$ is a result. ${ }^{25} \mathrm{High} M y c$ protein expression can occur through alternative mechanisms, including amplification, or in the absence of $M y c$ gene abnormalities. A large retrospective study found that $M y c$ translocations with $\mathrm{Bcl} 2$ translocation or TP53 mutation, but not $B c l 6$ translocation, were associated with significantly worse outcomes than $M y c$ rearrangement alone, and patients with concurrent mutation of TP53 had significantly the worst outcomes (Fig 2). ${ }^{26}$

One approach suggested to overcome highly proliferative lymphomas' resistance to R-CHOP is the DA-EPOCH-R (dose-adjusted etoposide, prednisolone, cyclophosphamide, doxorubicin, vincristine and rituximab) infusional regimen. This is being tested in a randomised trial to determine whether it is superior to R-CHOP in DLBCL. ${ }^{27}$ A preliminary report suggested that patients with a $M y c$ rearrangement or double $\mathrm{Myc} / \mathrm{Bcl} 2$ hits may be as responsive to DA-EPOCH-R as non$M y c$ rearranged patients, something not seen with R-CHOP. ${ }^{28}$ The small groups of GCB-subtype with $M y c$ translocation 

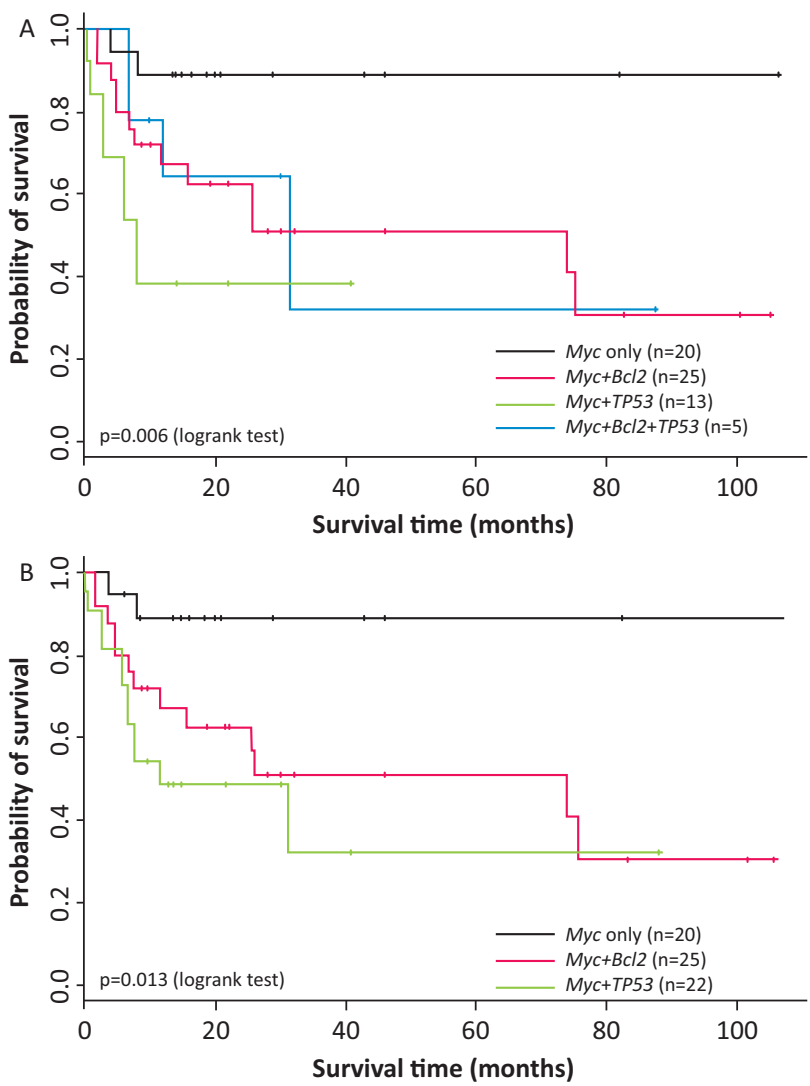

Fig 2. Impact of TP53 mutation and BCL2 translocation on overall survival of patients with Myc translocation positive DLBCL.

A - Myc translocation positive DLBCL are divided into four subgroups: Myc/ Bcl2 translocation/TP53 mutation, Myc translocation/TP53 mutation, $M y C / B C l 2$ translocation, and $M y c$ translocation only. The cases with the $M y c / B C 12 / T P 53$ triple-hit show the worst overall survival, followed by cases with the Myc/TP53 and those with the $M y c / B C l 2$ double-hit. The significant difference in overall survival between cases with the Myc/BCl2/TP53 triple-hit and those with isolated Myc translocation is also shown by Cox proportional hazards regression adjusted for age. B - The cases with TP53 mutation are combined together irrespective of their $B \mathrm{Cl} 2$ translocation status. The cases with TP53 mutation show a worse overall survival than those with an isolated Myc translocation, being statistically significant by Cox regression model adjusted for age ( $p=0.0059$ ). The cases with $B c / 2$ translocation also show a significantly worse overall survival than those with isolated Myc translocation by Cox regression model adjusted for age $(p=0.019)$. $D L B C L=$ diffuse large B-cell lymphoma. Reproduced from Clipson et al. ${ }^{26}$

or the ABC-subtype with $M y c$ and Bcl2 co-expression may represent examples by which $\mathrm{COO}$ and genetic abnormalities may be combined to select treatment that differs from the standard R-CHOP. Further work is needed to test whether such biomarkers might identify groups for which other types of treatment are superior to R-CHOP.

$M y c, B c l 2$ and $B c l 6$ could be considered attractive targets for specific therapy in DLBCL. Myc overexpressing lymphomas commonly show PIM1 co-expression, which is a co-activator of Myc. PIM1 mutations are more common in the ABC subtype, present in around $20 \%$ of cases. ${ }^{15}$ PIM1 inhibitors have shown evidence of effect in pre-clinical studies. Venetoclax, a $\mathrm{BH} 3$ mimetic that acts as a $\mathrm{Bcl} 2$ inhibitor, has demonstrated promising efficacy in relapsed and refractory DLBCL; however, the responses are not sustained, suggesting that $\mathrm{BH} 3$-mimetics may be most useful in combination therapy. ${ }^{29}$ A phase I study using BET bromodomain inhibitors (BETi), which reduce Myc transcripts, has shown responses in recurrent DLBCL. ${ }^{6}$ In pre-clinical work, resistance to BETi can be predicted by apoptotic network signal regulators' expressions, including $B c l 2$. While some resistant cell lines appear to escape apoptosis through upregulation of $B c l 2$, when $B c l 2$ inhibitors such as venetoclax are used these cell lines show growth inhibition. ${ }^{30}$ However, the redundancy of the signalling pathways does permit DLBCL cells to switch oncogenic addiction between different driver genes, indicating the need for small molecule drug combinations as a means to avoid the emergence of resistance. $^{31}$

\section{Conclusions}

Advances in our understanding of DLBCL biology have identified both predictive and prognostic biomarkers. Next-generation sequencing and GEP have identified marked heterogeneity between cases but immunochemotherapyresistance remains challenging to predict. Future work to prospectively and longitudinally map gene expression profile and mutations is needed to better understand DLBCL through correlation with the treatment response. We have an opportunity to incorporate novel agents into the armamentarium with their efficacy enhanced by molecular pre-selection. We anticipate that it will become increasingly necessary to incorporate diagnostic molecular phenotyping into routine clinical practice. Progress in DLBCL is likely to come through appreciation and targeting of heterogeneous subtypes. There are early phase trials that find that, while many patients with relapsed/refractory DLBCL do not respond to novel agents, there are some responders who attain excellent remissions. One method to develop effective approaches will be to use precision therapeutics at relapse according to driver mutation and $\mathrm{COO}$ to predict and test response. In much the same way that immunochemotherapy improved outcomes through combination therapy, so novel small molecule drugs given in combination seem the most promising approach. Where R-CHOP fails, we hope that these new approaches can succeed by providing personalised medicine using precision diagnostics to guide new treatment paradigms.

\section{Conflicts of interest}

PJ received research funding from Janssen-Cilag and Epizyme, and honoraria from Bristol-Myers Squibb, Pfizer, Takeda, BoehringerIngelheim and Celgene. TC has no conflicts of interest to declare

\section{References}

1 Coffier B, Thieblemont C, Van Den Neste E et al. Long-term outcome of patients in the LNH-98.5 trial, the first randomized study comparing R-CHOP to standard CHOP chemotherapy in DLBCL patients: a study by the GELA. Blood 2010;116:2040-5.

2 Grisselbrecht C, Glass B, Singh Gill D et al. Salvage regimens with autologous transplantation for relapsed large B-cell lymphoma in the rituximab era. J Clin Oncol 2010;28:4184-90.

3 Copie-Bergman C, Plonquet A, Alonso M et al. MAL Expression in Lymphoid Cells: Further Evidence for MAL as a Distinct Molecular 
Marker of Primary Mediastinal Large B-Cell Lymphomas. Mod Pathol 2002;15:1172-80.

4 Roschewski M, Staudt L, Wilson W. Diffuse large B-cell lymphomatreatment approaches in the molecular era. Nat Rev Clin Oncol 2014;11:12-23.

5 Hu S, Xu-Monette Z, Tzankov A et al. MYC/BCL2 protein coexpression contributes to the inferior survival of activated B-cell subtype of diffuse large B-cell lymphoma and demonstrates high-risk gene expression signatures: a report from The International DLBCL Rituximab-CHOP Consortium Program. Blood 2013;121:4021-31.

6 Thieblemont C, Briere J, Mounier N et al. The germinal center/ activated B-cell subclassification has a prognostic impact for response to salvage therapy in relapsed/refractory diffuse large B-cell lymphoma: a bio-CORAL study. J Clin Oncol 2011;29:407987.

7 Johnson PW, Davies A, Jack AIII. Applying molecular phenotyping in practice. Haematol Oncol 2013;31(Suppl 1):29-32.

8 Care MA, Barrans S, Worrillow L et al. A microarray platformindependent classification tool for cell of origin class allows comparative analysis of gene expression in diffuse large B-cell lymphoma. PloS One 2013;8:e55895.

9 Choi WL, Weisenburger D, Greiner T et al. A new immunostain algorithm classifies diffuse large B-cell lymphoma into molecular subtypes with high accuracy. Clin Cancer Res 2009;15:5494-502.

10 Wilson WH, Young R, Schmitz R et al. Targetting B cell receptor signaling with ibrutinib in diffuse large B cell lymphoma. Nat Med 2015;21:922-6.

11 Lam L, Davis R, Ngo V. Compensatory IKKalpha activation of classical NF-kappaB signaling during IKKbeta inhibition identified by an RNA interference sensitization screen. Proc Natl Acad Sci USA 2008;105:20798-803.

12 Dunleavy K, Pittaluga S, Czuczman M et al. Differential efficacy of bortezomib plus chemotherapy within molecular subtypes of diffuse large B-cell lymphoma. Blood 2009;113:6069-76.

13 Davies AJ, Caddy J, Maishman T et al. A prospective randomised trial of targeted therapy for diffuse large B-cell lymphoma (DLBCL) based upon real-time gene expression profiling: the Remodl-B study of the UK NCRI and SAKK lymphoma groups (ISRCTN51837425). Blood 2015;126:812.

14 Nowakowski GS, LaPlant B, Macon WR et al. Lenalidomide combined with R-CHOP overcomes negative prognostic impact of nongerminal genter B-cell phenotype in newly diagnosed diffuse large B-cell lymphoma: a phase II study. J Clin Oncol 2015;33:251-7.

15 Dubois S, Viailly P, Mareschai S et al. Next generation sequencing in diffuse large B-cell lymphoma highlights molecular divergence and therapeutic opportunities: a LYSA study. Clin Cancer Res 2016;22:2919-28.

16 Morin RD, Johnson NA, Severson TM et al. Somatic mutations altering EZH2 (Tyr641) in follicular and diffuse large B-cell lymphomas of germinal-center origin. Nat Genet 2010;42:181-5.

17 Zhou Z, Gao J, Popovic R et al. Strong expression of EZH2 and accumulation of trimethylated H3K27 in diffuse large B-cell lymphoma independent of cell of origin and EZH2 codon 641 mutation. Leuk Lymphoma 2015;56:2895-901.
18 McCabe MT, Ott HM, Korenchuk S et al. EZH2 inhibition as a therapeutic strategy for lymphoma with EZH2-activating mutations. Nature 2012;492:108-12.

19 Béguelin W, Popovic R, Teater $\mathrm{M}$ et al. EZH2 is required for germinal center formation and somatic EZH2 mutations promote lymphoid transformation. Cancer Cell 2013;23:677-92.

20 Ribrag V, Soria JC, Michot JM et al. A phase I study of tametostat (EPZ-6438), an inhibitor of EZH2: preliminary safety and activity in relapsed or refractory NHL patients. Blood 2015;126:473.

21 Barrans S, Crouch S, Smith A et al. Rearrangement of MYC is associated with poor prognosis in patients with diffuse large B-cell lymphoma treated in the era of rituximab. J Clin Oncol 2010;28:3360-5.

22 Cuccuini W, Briere J, Mounier N et al. MYC+ diffuse large B-cell lymphoma is not salvaged by classical R-ICE or R-DHAP followed by BEAM plus autologous stem cell transplantation. Blood 2012;119:4619-24.

23 Aukema SM, Siebert R, Schuuring E et al. Double-hit B-cell lymphomas. Blood 2011;117:2319-31.

24 Iqbal J, Sanger WJ, Horsman DE et al. BCL2 translocation defines a unique tumour subset within the germinal center B-cell-like diffuse large B-cell lymphoma. Am J Pathol 2004;165:159-66.

25 Copie-Bergman C, Cuilliere-Dartigues P, Baia M et al. MYC-IG rearrangements are negative predictors of survival in DLBCL patients treated with immunochemotherapy: a GELA/LYSA study. Blood 2015;126:2466-74.

26 Clipson A, Barrans S, Zeng N et al. The prognosis of MYC translocation positive diffuse large B cell lymphoma depends on the second hit. J Pathol Clin Res 2015;1:125-33.

27 Wilson WH, Jung SH, Porcu P et al. A Cancer and Leukemia Group B multi-center study of DA-EPOCH-rituximab in untreated diffuse large B-cell lymphoma with analysis of outcome by molecular subtype. Haematologica 2012;97:758-65.

28 Dunleavy K, Fanale M, LaCasce A et al. Preliminary report of a multicenter prospective phase II study of DA-EPOCH-R in MYCrearranged aggressive B-cell lymphoma. Blood 2014;124:395.

29 Davids MS, Seymour JF, Gerecitano JF et al. Phase I study of ABT199 (GDC-0199) in patients with relapsed/refractory non-Hodgkin lymphoma: responses observed in diffuse large B-cell (DLBCL) and follicular lymphoma (FL) at higher cohort doses. Clin Adv Hematol Oncol 2014;12(Suppl 16):18-9.

30 Conery AR, Centore RC, Spillane KL et al. Preclinical anticancer efficacy of BET bromodomain inhibitors is determined by the apoptotic response. Cancer Res 2016;76:1313-9.

31 Dupont T, Yang SN, Patel J et al. Selective targeting of BCL6 induces oncogene addiction switching to BCL2 in B-cell lymphoma. Oncotarget 2016;7:3520-32.

Address for correspondence: Dr T Cummin, Somers Cancer Research Building MP824, Southampton General Hospital, Tremona Road, Southampton S016 6YD, UK.

Email: tcummin@gmail.com 